\title{
Evaluación de la gestión económico-financiera en microempresas
}

\section{mexicanas}

\section{Evaluation of the economic and financial management in Mexican microenterprises}

Revista FIR, FAEDPYME International Review // Vol. 5 Nํ 8 // enero - junio de 2016 // pp. 20-31 // e-ISSN: 2255-078X

\section{Malena Portal Boza}

Profesora Tiempo Completo, Facultad de Contaduría y Administración, Universidad Autónoma de Baja California, Campus Tijuana, B.C., México.

Doctorado en Ciencias Económicas, Facultad de Economía y Relaciones Internacionales, Universidad Autónoma de Baja California (UABC) Campus Tijuana. Maestría en Dirección de Empresas, Licenciatura en Contabilidad y Finanzas, Universidad Central "Martha Abreu” de las Villas, Cuba. Coordinadora del Área de Finanzas, Facultad de Contaduría y Administración de la Universidad Autónoma de Baja California (UABC).

E-mail: mportal@uabc.edu.mx

\section{Duniesky Feitó Madrigal}

Profesor Tiempo Completo de la Facultad de Contaduría y Administración de la Universidad Autónoma de Baja California (UABC) Campus Tijuana, B.C., México. Doctorado en Ciencias Económicas, UABC. Maestría en Administración de Negocios, Ingeniero Industrial, Universidad Central “Martha Abreu” de las Villas, Cuba. Coordinador de Carrera en la Licenciatura en Administración de Empresas Facultad de Contaduría y Administración de la Universidad Autónoma de Baja California (UABC).

E-mail: duniesky.feito.madrigal@uabc.edu.mx

\section{Blanca Estela Bernal Escoto}

Profesora-Investigadora de Tiempo Completo de la Facultad de Contaduría y Administración de la Universidad Autónoma de Baja California, Campus Tijuana, B.C., México. Doctorado en Planeación Estratégica para la Mejora del Desempeño, Instituto Tecnológico de Sonora. Maestría y Licenciatura, Administración, UABC. Responsable del Programa de Formación y Actualización Docente de la Facultad de Contaduría y Administración de la Universidad Autónoma de Baja California y coordinadora del área de Mercadotecnia.

E-mail: blancab@uabc.edu.mx

Fecha de recepción: 2016-01-27

Fecha de aprobación: 2016-06-06

\section{Resumen.}

Las características que exhiben las microempresas mexicanas en la actualidad dan evidencian de las problemáticas que estas enfrentan en cuanto a la gestión de sus actividades, donde se percibe el protagonismo de los elementos económicos-financieros. Aun cuando la temática haya sido abordada en investigaciones precedentes la aportación de este estudio radica en evaluar de manera integrada el nivel de gestión económico-financiera (GEF) en las microempresas y el impacto sobre los resultados empresariales. Para ello se construye un índice a partir de la metodología de indicadores sintéticos aplicando el análisis factorial por componentes principales, acompañada de ejercicios de regresión lineal. Los resultados obtenidos confirman la presencia de elementos de GEF en las microempresas estudiadas. Por otra parte, se encontró que en la medida en que se incorporen elementos de GEF en sus actividades, el impacto en sus resultados empresariales será positivo. Estas observaciones constituyen la antesala para la generación de políticas públicas en busca de establecer estrategias de mejora para este tipo de empresas, además de que propicia investigaciones futuras para realizar el análisis pero por sectores de actividad económica. 


\section{Abstract.}

The exhibit features Mexican microenterprises today give evidence of the problems that they face in terms of managing their activities, where the role of economic-financial elements is perceived. Although the issue has been addressed in previous studies the contribution of this study is to evaluate in an integrated manner the level of economic and financial management (GEF) in micro and impact on business results. To do an index is constructed from indicators synthetic methodology using the principal components factor analysis, accompanied by linear regression exercises. The results confirm the presence of elements of GEF in micro studied. Moreover, it was found that the extent to which GEF elements are incorporated in its activities, the impact on their business results will be positive. These observations constitute the prelude to the creation of public policies seeking to establish improvement strategies for such companies, besides that fosters future research for analysis but by sectors of economic activity.

Keywords: economic and financial management, microenterprises. 


\section{Introducción}

A nivel internacional, los estudios realizados a las microempresas han cobrado un protagonismo importante dentro de la gama de investigaciones que se vienen desarrollando en diversas áreas del conocimiento, sobre todo vinculadas a temáticas económicas y administrativas. Gran parte de estos estudios se concentran en Latinoamérica por la representatividad que tiene este tipo de empresas dentro de las estructuras empresariales de la región, donde México tiene una destacada participación.

En las últimas décadas, la economía mexicana ha experimentado cambios en su estructura empresarial. La participación de las microempresas en las estructuras empresariales se evidencia en las estadísticas de los censos económicos, donde entre el 2004 y el 2014 este rubro representaba aproximadamente el $95 \%$ del total de las compañías existentes y empleaban al $39.7 \%$ del total de trabajadores.' Las estadísticas reflejan la importancia que tienen las microempresas dentro de la economía mexicana sobre todo en la contribución a la generación de empleos, que se ha convertido en alternativas de solución para la sostenibilidad de diversas familias. De ahí el incremento en los estudios dirigidos hacia este sector, los cuales han cobrado un mayor auge debido a la necesidad de conocer cómo funcionan, cuáles son sus problemáticas y limitaciones. Pavón (2010) hace referencia a estadísticas que reflejan la alta tasa de mortalidad de las micro, pequeñas y medianas empresas en México, menciona que de las 200 mil empresas que anualmente abren sus puertas en México cada año, solamente 35 mil sobreviven dos años más tarde, en el caso específico de las microempresas, de cada diez que surgen, ocho desaparecen al año. Por su parte, Rodríguez (2010) hace un resumen de varias investigaciones realizadas sobre las causas del fracaso en las microempresas, mencionando la presencia de problemas en cuanto a la gestión de sus actividades donde los elementos económicos-financieros no están excluidos. El autor concluye que en la práctica, los propietarios fracasan y no es debido a razones técnicas, en el sentido de que no tengan conocimientos sobre la actividad que realizan, sino más bien, por tener desconocimiento en contabilidad y finanzas, administración, falta de controles económicos-financieros, carencia de capital suficiente, entre otros.

La gestión económico-financiera (GEF) constituye una actividad de gran importancia para cualquier tipo de empresa independientemente de su tamaño, y en el caso de las microempresas mexicanas está considerada como una de las causantes asociadas a su poca permanencia en los mercados, ya que esta actividad tiene un vínculo fuerte con decisiones que determinan el futuro de las mismas. Simpson (2011) argumenta que la gestión económico-financiera se ocupa de la asignación eficiente de capital, establecimiento y estudio de precios, flujos de efectivo, costos de capital y sobre todo, que el capital siempre se invierta de manera tal que produzca la mejor rentabilidad ajustada al riesgo. Por lo que resulta de especial interés contar con investigaciones que analicen, evalúen y arrojen información conclusiva acerca de cómo se manifiesta esta actividad en la gestión de las microempresas y cuál es el efecto que tiene sobre sus resultados empresariales.

La revisión de la literatura sobre esta temática de análisis, arrojó la presencia de estudios empíricos que han abordado algunos de los factores que forman parte de la gestión económico-financiera, un poco más inclinados a la gestión financiera que a la económica. No obstante, a pesar de la presencia de estos estudios, no se encontró evidencia de investigaciones que realizaran una evaluación de la GEF vista como un indicador único e integrador, y el impacto que tiene en los resultados que obtienen este tipo de empresas.

Teniendo en cuenta los argumentos expuestos anteriormente, el presente estudio tiene como objetivo general evaluar el nivel de GEF en las microempresas mexicanas y a su vez medir el efecto que tiene sobre los resultados empresariales. Para ello se propone utilizar la metodología de construcción de indicadores sintéticos para la creación del índice, y una vez calculado evaluar el nivel de gestión que manifiestan las microempresas estudiadas. Posteriormente se realizan varios ejercicios de regresión lineal para determinar el efecto que tiene la gestión económico-financiera expresada a través del índice, sobre los resultados empresariales de este tipo de empresas, primero de manera general y luego con el índice distribuido en intervalos de clase. La información que se utiliza proviene de la Encuesta Nacional de Microempresas 2012, la cual es la investigación más actualizada con la que se cuenta a partir del último censo realizado y publicado en México.

\section{Revisión de literatura y planteamiento de hipótesis.}

A lo largo de la historia las concepciones sobre la gestión han ido en evolución, por la propia necesidad de adaptar las teorías a la realidad empírica y por la importancia que ha ido alcanzando no solo en el ámbito empresarial, sino también en nuestra vida social y económica. Es por ello que se continúan desarrollando nuevos conceptos, ideas y opiniones al respecto. Algunos de los autores que se dedicaron al estudio teórico del concepto fueron Henry Fayol, Walther Rathenau, Mary Parker Follet y H.L.Gantt, Frederic Winslow Taylor, y Peter Drucker, entre otros. Amat (1992) afirma que la gestión está caracterizada por una visión más amplia de las posibilidades reales de una organización para resolver determinada situación o arribar a un fin determinado. Cuando se habla de sistemas de gestión, se hace referencia esencialmente a los procesos racionales de gestión de una determinada actividad $y$ a los elementos que confluyen en esos procesos para ha- 
cerlos posibles. Por tanto, el proceso de gestión abarca todas las áreas que comprende una organización, debido a que todas las actividades están involucradas de alguna forma en el cumplimiento de los objetivos de la gestión empresarial. Una de las actividades esenciales en la gestión de las organizaciones es precisamente la económico-financiera. Para Van (1995), la gestión empresarial no se puede entender separada de la gestión financiera y menos de la gestión económica. En la revisión de la literatura, la mayoría de los estudios que se encuentran, están presentados o enfocados en función de los elementos que componen la actividad económico-financiera. Es decir, en los modelos, la gestión económico-financiera se muestra a través de las decisiones de financiamiento, gestión de inversiones, contabilidad de gestión, o cuando son estudios en temas más específicos se trabajan a partir de la gestión de sus componentes como por ejemplo: gestión de Inventarios, gestión de cuentas por cobrar, gestión de proveedores y contratación, gestión de las cuentas por pagar, gestión de presupuestos, entre otros.

En términos teóricos la gestión económica es la encargada de llevar a vía de hecho toda la actividad de una organización con eficiencia y eficacia, con vistas a que las empresas obtengan los resultados esperados. Es una terminología que se relaciona con la obtención de rentabilidad en las empresas. Por su parte, la gestión financiera se concentra en las decisiones de inversión y financiamiento, es decir, de dónde obtener los recursos financieros necesarios para garantizar las inversiones y la operatividad de las empresas, pero además garantizar un uso eficiente de los mismos, analizando costos asociados a las cuotas mensuales de intereses, montos requeridos, establecer prioridades, plazos de pago y analizar niveles de riesgos. En la literatura se encuentran diversidad de criterios y conceptualizaciones teóricas acerca de la gestión económico-financiera que presentan sus puntos de coincidencia.

Santiesteban, Fuentes, Leyva y Lozada (2009), manifiestan que la gestión económica a través de los niveles de rentabilidad constituye un indicador que evalúa de forma sostenida y rigurosa los resultados de una organización. Lawrence (2007) expresa que la rentabilidad se basa en la relación entre ingresos y costos generados por el uso de los activos de la empresa en actividades productivas. Representa toda acción económica en la que se movilizan medios y recursos materiales, humanos y financieros, con el fin de obtener ciertos resultados. Autores que se ocuparon del estudio de la gestión económico-financiera hacen referencia a las funciones que incluye esta actividad como Ochoa y Saldívar del Ángel (2012, p. 4) los cuales manifiestan: "...las Finanzas se vinculan a la Economía en relación a la asignación de recursos y la utilización de información proveniente de la contabilidad...", además de que "...las decisiones de financiamiento implican estudiar la manera en que la empresa adquiere los recursos necesarios para llevar a cabo las decisiones de inversión..." (2012, p. 7). Block y Hirt (2013, p. 4) argumentan: "los empresarios dependen de la administración financiera para hacer un uso eficiente y rentable de los recursos de que dispone" y además exponen que dentro de las funciones financieras se incluyen administrar el flujo de efectivo, comprar materiales y equipo, plantas, adquisición de capital y tomar decisiones financieras en cuanto a factibilidad y rentabilidad del flujo continuo de nuevos productos.

Por otra parte Paramasivan y Subramanian (2009) proponen algunas funciones importantes dentro de la gestión económico-financiera como, estimar cuánto se necesita para la adquisición de activos fijos, seleccionar cuidadosamente las mejores alternativas de inversión y considerar la rentabilidad razonable y estable de la inversión.

De las definiciones mencionadas se pueden seleccionar elementos comunes que apuntan hacia una coincidencia entre gran parte de los autores. Estamos refiriéndonos a que la gestión económico-financiera, concierne a las decisiones de inversión y financiación de la empresa como elementos protagónicos, incluyendo la selección de las fuentes de financiamiento de donde provienen los fondos demandados y sobre todo, que los recursos invertidos generen rendimientos para la empresa, además de que lleva aparejado la utilización de la información contable para la toma de decisiones y el estudio de los costos asociados. La meta de cualquier empresa independientemente de su tamaño es la maximización de utilidades y la creación de riqueza para sus dueños, por ello es importante que las empresas manejen niveles altos en su gestión económico-financiera que les permita obtener resultados positivos. En relación a ello Ross, Westerfield y Jaffe (2012, p. 11) manifiestan que el "...evitar dificultades financieras, la quiebra, derrotar a la competencia, maximizar ventas y minimizar costos...", son metas que se relacionan con la rentabilidad y se pueden lograr llevando a cabo estrategias como por ejemplo reducción de precios, relajación de términos de crédito, solicitar fondos en préstamos, entre otras. Keat y Young (2004) consideran que la gestión económico-financiera cuando se ejerce de manera adecuada, sobre todo la administración financiera, puede hacer la diferencia entre la supervivencia de una pequeña empresa y su desaparición, ya que no solo se debe garantizar la generación de utilidades sino también, flujos de efectivo que sean positivos y suficientes.

Por tanto, tomando en consideración el objetivo de esta investigación resulta necesario hacer alusión a cómo se ve representado en las microempresas la gestión económicofinanciera. En este sentido las microempresas, a pesar de tener las características de ser negocios pequeños, aperturados con capital mínimo, donde sus propietarios y trabajadores tienen poco conocimiento en herramientas y prácticas de gestión y administración y poseer varias limitaciones en sus operaciones, no dejan de realizar actividades de gestión económico-financiera. Estas microempresas establecen sus necesidades de recursos y realizan inversiones para garantizar su operatividad, sobre todo en materias primas y materiales, herramientas, equipamiento, transporte, entre otros. También determinan el precio de sus productos y servicios, 
sus costos y gastos para tratar de garantizar al menos, un nivel mínimo de rentabilidad de su negocio, una utilidad que les permita sostener a sus familias y mantenerse en el mercado. Como parte de sus actividades de gestión buscan estrategias para atraer clientes, o para diferenciarse de la competencia ya que una de las peculiaridades de este tipo de empresas es que crean relaciones estrechas con la comunidad y por otra parte, buscan las opciones que tienen disponible para obtener los recursos financieros necesarios, es decir, las fuentes de financiamiento que tienen a su alcance, valoran los montos de financiamiento que necesitan para cumplir con sus demandas y también los costos que se derivan de la obtención de los mismos. En relación a ello Brealey y Myers (2010) opinan que en empresas grandes las funciones relacionadas sobre todo con la gestión financiera se pueden dividir entre el Director de finanzas, el tesorero y el contralor, pero en el caso de las pequeñas empresas por lo general es una persona la que se ocupa de toda la actividad de gestión, y las funciones que se manifiestan con una mayor representatividad en este tipo de empresas son las que se asocian al manejo del efectivo, conseguir capital y las relaciones con los bancos.

En los fundamentos teóricos de la gestión económicofinanciera se encuentra también, como se mencionó anteriormente, la presencia de la contabilidad como un elemento importante para la obtención de información necesaria para la toma de decisiones. Autores como Block y Hirt (2013, p. 5) plantean que "la Contabilidad es el lenguaje de las finanzas porque proporciona datos financieros a través de los estados que se emiten...", la cual se utiliza para "... poder asignar los recursos de la empresa de tal forma que al largo plazo generen el mayor rendimiento posible..." Si bien es cierto que la mayoría de los estudios realizados reflejan que las microempresas se caracterizan por llevar una contabilidad no profesionalizada, no significa que en estas empresas no se puedan aplicar herramientas contables y de control como la utilización de talonarios de cheques, cintas de cajas registradoras, llevar algún tipo de registro en apuntes personales, realizar arqueos al efectivo, establecer qué tipo de documentos primarios se utilizarán para el registro de las transacciones como comprobantes o notas de ventas, facturas, registros de proveedores y clientes, entre otros.

Cuando se consultan los estudios empíricos realizados en la temática, la evidencia refleja que es posible identificar y representar la gestión económico-financiera en las micro y pequeñas empresas con una mayor presencia de enfoques dirigidos hacia la gestión financiera y en menor medida la gestión económica. Como parte de estos estudios se encuentran algunos a nivel internacional como el estudio realizado por Mazzarol, Reboud y Clark (2015) donde se dedicaron a examinar las prácticas de gestión financiera en una muestra de pequeñas y medianas empresas en Australia y Singapur, así como las percepciones de los propietarios y el impacto de su comportamiento en el desempeño de las PYME, encontrándose que existían algunas irregularidades asociadas sobre todo al control y supervisión de sus finanzas y al establecimiento de precios, insuficiente capital de trabajo disponible para el crecimiento, ausencia de endeudamiento bancario excesivo o incluso la financiación de capital, que no sea de la familia o amigos. La mayoría de los propietarios preferían financiar sus negocios en sus inicios de los ahorros personales y luego de los beneficios retenidos. Estos autores concluyeron que la mayoría de los propietarios tenían una débil capacidad de gestión financiera y sus empresas carecían de sistemas sofisticados para controlar las finanzas, mientras que la mayoría contaba con paquetes de software para la contabilidad, pero que estos fueron utilizados para la contabilidad financiera a efectos fiscales en lugar de para las tareas de contabilidad de gestión. Otro estudio identificado en Sudáfrica, realizado por Mungal y Garbharran (2014) demuestran en sus resultados una correlación entre la rentabilidad del negocio y la aplicación de prácticas de gestión financieras principalmente en la gestión del efectivo. Se argumenta que una deficiente gestión de caja es un factor que contribuye a una menor rentabilidad y afecta la sostenibilidad de las pequeñas empresas, además el estudio confirmó que la restricción a un préstamo incide en el rendimiento financiero de este tipo de empresas. Por otra parte, Dahmen y Rodríguez (2014) presentaron una investigación realizada con el apoyo del Centro de Desarrollo de Pequeñas Empresas de la Universidad del Sur de Florida, donde el objetivo principal constituyó la evaluación de la gestión económico-financiera de un grupo de pequeñas empresas a través de la aplicación de herramientas de análisis financiero y pudieron detectar que el $86 \%$ de la muestra presentó dificultades en su gestión principalmente asociadas a la falta de comprensión y uso de la información económico-financiera por parte de los propietarios para la toma de decisiones. Otro de los estudios empíricos realizados a nivel internacional lo constituye el trabajo presentado por Solomon (2014) sobre la evaluación de las prácticas de gestión financiera en 70 pequeñas empresas, encontrando que la aplicación de estas es muy débil, especialmente en las áreas de planificación financiera, análisis y control, gestión de capital de trabajo y las decisiones de inversión. También Rathnasiri (2015) se basó en el principio de que las prácticas de gestión financiera constituye una de las herramientas para mejorar y aumentar la rentabilidad y en función de ello investigó en una muestra de PYMES en Sri Lanka las posibles causas que generan las diferencias en la adopción de herramientas de gestión financiera, evaluando aspectos tales como forma jurídica, el tamaño y la edad de la empresa, el nivel de educación del propietario, la ubicación y el apalancamiento.

En el caso de Latinoamérica Correa y Jaramillo (2007) plantean una serie de lineamientos generales para la gestión financiera en las pequeñas empresas en Colombia incluyendo herramientas para la planeación, el control y la toma de decisiones financieras. Escobar, Arias y Portilla (2009) 
proponen un modelo para medir la situación financiera en PyMES en Colombia. Por su parte, Mariño, Gladys y Medina (2009), realizaron una comparación de la forma como se ejerce la administración financiera en Estados Unidos, México, España y Colombia, y además analizaron la brecha existente entre la teoría y la práctica de la administración financiera, a partir del contraste de la teoría propuesta por diferentes autores expertos en el tema. Es válido destacar que en este estudio se encontraron algunos resultados interesantes que se vinculan mucho al tema de la presente investigación, como que el $47.8 \%$ de las MIPyME no utilizan herramientas financieras para la toma de decisiones, el $45 \%$ de las empresas llevan la contabilidad en libros, seguido muy de cerca por facturas de compra y venta, mientras que un porcentaje muy pequeño el $2 \%$, perteneciente al sector comercio al por mayor, posee algún tipo de software contable. Además se encontró que en promedio, en el $54 \%$ de las empresas, quien maneja el área financiera es el dueño y de éstos el $52 \%$ son bachilleres y en porcentajes muy pequeños existe la presencia de un contador o un auxiliar contable.

Para el caso de México se encuentra el estudio de Ramírez, Mungaray y Guzmán (2009), donde en sus estudios determinaron la existencia de restricciones de liquidez para las microempresas analizadas $y$ al acceso a fuentes de financiamiento informales. Aguilar, Ramírez y Barrón (2007) realizaron una investigación en las microempresas de la frontera norte y demostraron que existe relación entre algunos elementos de la gestión económico-financiera incluidos en su estudio y las mejoras en los resultados empresariales, por ejemplo el hecho de que los proveedores otorguen o no crédito a la microempresa y el tipo de contabilidad utilizada en el negocio, planeación o no de las compras de insumos, con el hecho de mantener o no inventarios y el nivel diario de ventas, la necesidad o no de financiamiento y el tipo de compras que realiza la empresa, entre otros. También se encuentra el estudio realizado por Mungaray, Ocegueda, Ledezma, Ramírez, Ramírez y Alcalá (2007) en más de 150 microempresas marginadas de los estados de Baja California y Nayarit, donde a través de la aplicación de metodología tradicional de las técnicas de evaluación económica, observaron que los empresarios suelen desconocer el nivel de rentabilidad de su negocio y no tienen información de otros parámetros que les permitan optar por invertir en otro segmento del mercado.

Tomando en consideración los objetivos del estudio, así como la revisión teórica y empírica acerca del tema, se formulan las siguientes hipótesis que apoyarán a la solución de la problemática que se presenta:

H1: El nivel de gestión económico-financiera que se lleva a cabo en las microempresas mexicanas se mide a partir del análisis de las decisiones de inversión y financiación, unido a la utilización de registros y controles contables como fuente de información oportuna que contribuya al rendimiento de los recursos.

H2: En la medida en que las microempresas operen con mayores niveles en su gestión económico-financiera, el impacto que se creará en sus resultados empresariales será positivo.

\section{Metodología}

La información utilizada para el estudio empírico se obtuvo a partir del procesamiento de la base de datos proveniente de la Encuesta Nacional de Micronegocios (ENAMIN 2012) la cual constituye un trabajo conjunto de la Secretaría del Trabajo y Previsión Social (STPS) y del Instituto Nacional de Estadística y Geografía (INEGI) en México. Esta base de datos es la que se maneja en el país para obtener información sobre microempresas a nivel nacional y tiene como objetivo ofrecer información estadística, representativa, sobre las principales características económicas de los micronegocios y sobre las condiciones laborales de la población involucrada en ellos.

En base a los fundamentos teóricos y empíricos que sustentan el estudio se pudo establecer en el apartado anterior, que para el caso de las microempresas los elementos de gestión económico-financiera que se ven representados se vinculan a decisiones de inversión, financiación y el uso de controles económicos contables que contribuyan a que los recursos gestionados generen los rendimientos que se esperan. En función de esto se realizó un examen detallado de las preguntas de la encuesta utilizada para seleccionar aquellas que pudieran formar parte del análisis propuesto. En este sentido fueron determinadas como variables proxys representativas para el análisis las siguientes interrogantes: cómo llevan la actividad contable (P-37), qué tipo de comprobantes de venta expide a sus clientes (P-38), cómo fi c el precio de sus mercancías o servicios (P-48), qué empresas invirtieron en el último año y el monto de la inversión (P-56), de dónde provino principalmente el dinero para iniciar el negocio ( $\mathrm{P}-80)$, de dónde provino el préstamo más reciente (P-83), de las preguntas 86 a la 89 permiten analizar el monto del préstamo que se recibió, cuánto paga al mes por el préstamo, qué plazo le dieron para pagar y en qué invirtió el financiamiento obtenido. Estas variables serán las que se utilizarán para la obtención del índice de GEF a partir de la metodología de Análisis Factorial propuesta, a la cual le sigue un ejercicio de regresión para determinar el impacto del índice sobre los resultados empresariales como se menciona más adelante. En este ejercicio se tomará como variable dependiente la pregunta 69- cuánto le deja el negocio al mes como promedio, por considerarse la que más se aproxima a representar los resultados empresariales. Por su parte las variables independientes que forman parte del análisis, además del índice construido, están conformadas por las preguntas 52-horas totales trabajadas a la semana y 57- valor de los activos, como proxys ${ }^{2}$ de trabajo y capital

2. Es una terminología utilizada para nombrar variables aproximadas a las variables objeto de análisis. 
respectivamente. También se incluye la información sobre sexo y edad, para ser utilizadas como variables de control en el modelo de regresión que se estima. Cabe señalar que las variables utilizadas son de tipos nominales, ordinales y de razón dependiendo del tipo de pregunta y la escala utilizada.

La muestra seleccionada para el estudio partió del procesamiento de la base de datos donde, al eliminar aquellas respuestas nulas que no contienen información, se logró un número total de observaciones de 2692 microempresas distribuidas entre los Estados que posee el territorio nacional mexicano, con una mayor representatividad del Estado de México, el Distrito Federal y Jalisco. En relación a las actividades económicas, el sector servicios es el que mayor representatividad tiene en la muestra estudiada con un total de 1275 establecimientos, seguidos del sector comercio con 951 y por último el sector manufactura con 466.

Como se mencionó al inicio, uno de los objetivos del presente estudio consiste en la medición del impacto que tiene la GEF en los resultados empresariales. Para ello se propone la creación de un índice sintético de gestión económico-financiera. La creación de índices sintéticos se ha convertido en una herramienta de gran aplicación en diversas áreas de estudio. Los Indicadores o Índices Sintéticos o Complejos se utilizan cuando el interés de analizar aisladamente indicadores particulares es limitado y se requiere elaborar un indicador de significado más amplio. Ejemplos de estos indicadores son: el Índice de Desarrollo Humano, la Esperanza de Vida al Nacer, el Índice de Precio de Clientes. (Suárez, 2003, p. 8)

Para la obtención del índice se propone la aplicación de la metodología basada en el análisis multivariante con la técnica de análisis factorial a través de Componentes Principales (ACP). Es una metodología que ha sido ampliamente utilizada cuando se trata de construcción de índices de este tipo. Permite la extracción de los factores y la determinación del número de estos y consiste en estimar las puntuaciones factoriales mediante las puntuaciones tipificadas de las $k$ primeras componentes principales y la matriz de cargas factoriales mediante las correlaciones de las variables originales con dichas componentes. Ahora bien, esta metodología se basa en una serie de pasos: 1.Formulación del problema, 2.Cálculo y análisis de la matriz de correlación, 3.Extracción y determinación del número de factores, 4.Rotación de factores, 5.Validación del modelo, 6.Obtención e interpretación de las puntuaciones factoriales que serán utilizadas en las estimaciones y 7.Análisis posteriores (creación del índice y regresiones). Antes de aplicar la técnica de ACP recurrimos en el caso de la presente investigación, al análisis factorial de tipo exploratorio. El análisis exploratorio se caracteriza porque no se conocen a priori el número de factores y es en la aplicación empírica donde se determina este número. Ahora bien, uno de los requisitos que debe cumplirse para que la metodología pueda ser aplicada es que las variables estén altamente intercorrelacionadas. Para ello se utiliza la prueba de esfericidad de Bartlett y el índice Kaiser Meyer OIkin (KMO) como técnicas de validación. Una vez realizada la comprobación de idoneidad de la metodología se procede a la obtención y extracción de los factores, así como la determinación del número de estos y su ponderación, para luego confeccionar el índice de gestión económico-financiera (IGEF). Los valores que toman los componentes permiten conocer la participación que tiene cada microempresa dentro de cada factor extraído. De igual forma, para la construcción del índice sintético de gestión económico-financiera de todos los componentes se utilizará como ponderaciones, el porcentaje de varianza explicado por cada uno de ellos, lo cual se representa a través de la siguiente expresión:

$$
I G E F=\frac{\sum_{i=1}^{n} \operatorname{VAR}\left(p_{i}\right) * P_{i}}{\sum_{i=1}^{n} \operatorname{VAR}\left(P_{i}\right)}
$$

Donde: Donde $P i$ es el valor del componente para cada microempresa; $V A R(P i)$ es el porcentaje de varianza total que explica $P i$ y $n$ es el número de componentes extraídos. Cuando se construye un índice se enfrenta la condición de heterogeneidad de medida, por lo que para llevar a una misma escala de análisis el resultado de cada componente y poder interpretarlos, se hace necesario realizar una estandarización tomando como base los valores máximos o mínimos de cada indicador, lo cual se representa a través de la siguiente expresión:

$$
\left.V N_{i j}=\frac{V_{i j}-V_{\min (j)}}{V_{\max (j)}-V_{\min (j)}} 2\right)
$$

Siendo $V N_{i j}$ el valor estandarizado del componente $j$ para la microempresa $i, V_{i j}$ es el valor inicial del componente $j$ para la microempresa $j ; V_{\min (j)}$ es el valor mínimo que alcanza una microempresa en el componente $j ;$ y $V_{\max (j)}$ es el valor máximo que alcanza una microempresa en el componente $j$.

Este proceso de estandarización también conocido como normalización, nos permite hacer conclusiones acerca de los resultados obtenidos ya que la escala de valores se encontrará entre 0 y 1 , donde las microempresas que tengan valores cercanos a la unidad indicará un mejor IGEF y las que estén cercanas a 0 lo contrario. Para poder establecer estos criterios de agrupación se utilizará la metodología estadística que establece rangos de clase según los valores que alcanza cada microempresa dentro del índice estandarizado. Esta agrupación será utilizada para realizar el último paso del estudio, que es precisamente determinar el impacto que tiene el IGEF construido, en los resultados empresariales de las microempresas analizadas. Para ello se utilizará el Método de Regresión, que realiza la estimación mediante la metodología de los mínimos cuadrados. En el análisis de regresión lineal múltiple, se cuenta con un conjunto de variables independientes $X_{1^{\prime}} X_{2^{\prime}} \ldots X_{3^{\prime}}$ para estimar la variable dependiente $(\mathrm{Y})$. Se estimará una función de producción de tipo Cobb-Douglas ampliamente utilizada con la presencia 
de Capital (K), Factor trabajo (L) y (A) un residuo asociado a la productividad total de los factores, además se incluirá el IGEF determinado a través de las técnicas y las herramientas explicadas anteriormente. La función de producción establece un vínculo, una relación de dependencia entre producción y la totalidad de los medios empleados para realizarla partiendo de la siguiente formulación:

$$
Y i=F\left[e^{\alpha(i g e f, z)}, \beta 1, \beta 2, K i, L i\right]
$$

En esta formulación, el parámetro $\alpha$ (igef,z) constituye la constante o intercepto de la regresión. Las variables $\mathrm{Ki}$ y $\mathrm{Li}$ representan los factores capital y trabajo con sus parámetros asociados $\beta 1$ y $\beta 2$, que son los coeficientes de dichos factores respectivamente. La constante de regresión $\alpha$ (igef,z), se hace depender de la parte de la gestión económico-financiera en la microempresa atribuible al IGEF construido y de otros efectos de control, considerados explícitamente en el modelo (z). El residuo puede incluir otros efectos no explícitamente incorporados al modelo. Esta ecuación asume los factores, capital y trabajo, como esenciales para el proceso productivo en la empresa, mientras que la gestión económico-financiera se asocia con la eficiencia en el uso de los factores productivos por lo que se refleja en la productividad total de los factores (PTF). En esta investigación se toman las variables de capital y trabajo como se había mencionado anteriormente y en el caso de la PTF serán considerados los elementos de la gestión económico-financiera que estarán representados a través del IGEF. Además, serán incluidas las variables, sexo y edad, como variables de control que mejoran el ajuste del modelo. Es válido destacar que adicionalmente al análisis de regresión con el IGEF determinado, se incluyen al modelo en un análisis posterior, variables dummy que representarán los rangos de clases obtenidos y de esta forma poder comprobar cómo se comporta el impacto del índice a medida que se mueven los intervalos de clase.

\section{Resultados}

A través de la aplicación de la metodología propuesta primeramente se realizó una evaluación del supuesto de correlación entre las variables, con el fin de establecer si se justifica o no la aplicación del análisis factorial. Para ello nos basamos en el test de esfericidad de Bartlett y el índice KMO. El análisis de los test indica que en el caso del de Bartlett específicamente, se obtiene un valor de significancia menor a 0.05 por lo que se demuestra la existencia de correlación estadísticamente significativa entre las variables y en el caso del KMO el valor es de 0.631 , el cual se encuentra entre el rango aceptable para la aplicación del modelo $(0,75>$ $\mathrm{KMO} \geq 0,5)$. En esta primera evaluación, al aplicar los dos métodos anteriormente mencionados, se demuestra que se cumple el supuesto de correlación entre las variables por lo que es recomendable y se considera, que tiene sentido realizar el análisis factorial. Para el AFE, las variables utilizadas partieron de la revisión teórica sobre la problemática de estudio, las cuales quedaron definidas en el apartado anterior en base a las preguntas de la encuesta que fueron seleccionadas para representar los elementos de GEF en las microempresas estudiadas. Utilizando el programa SPSS, se procedió a la extracción y determinación del número de factores, su rotación y validación para determinar las variables que conformarán el índice de GEF. El cuadro 1 muestra los resultados arrojados.

La información nos confirma que es posible identificar componentes de GEF en las microempresas analizadas, integrados a través de 3 factores generados, los cuales agrupan elementos de contabilidad y registro, comprobante de ventas y el tiempo que le dieron para pagar el préstamo solicitado en el factor 1 (f1), cantidad invertida, monto del préstamo y cuánto paga al mes en el factor 2 (f2), fuentes de financiamiento tanto para iniciar el negocio como recibidas

\section{Cuadro 1. Matriz de componentes Rotados.}

\begin{tabular}{lccc}
\multicolumn{1}{c}{ Variables } & \multicolumn{3}{c}{ Componentes (factores) } \\
& $f 1$ & $f 2$ & $f 3$ \\
Cómo llevan la contabilidad (P-37) & $C 0.723$ & 0.316 & \\
Qué tipo de comprobantes de venta expide a sus clientes (P-38) & 0.714 & 0.361 & -0.321 \\
De dónde provino principalmente el dinero para iniciar el negocio (P-80) & & & 0.685 \\
De dónde provino el préstamo más reciente (P-83) & 0.438 & 0.646 \\
Monto de la Inversión (P-56) & & 0.476 & \\
Monto del préstamo (P-86) & & 0.631 & \\
Cuánto paga al mes por el préstamo (P-87) & 0.705 & \\
Plazo para pagar (P-88) & 0.707 & & -0.386 \\
En qué invirtió el financiamiento obtenido (P-89) & & & \\
Método de extracción: Análisis de componentes principales. & & & \\
Método de rotación: Normalización Varimax con Kaiser. & & & \\
Fuente: Adaptado de Flores et al. (2015). & & & \\
\end{tabular}


recientemente en el factor 3 ( $f 3$ ). Cabe señalar que del total de variables seleccionadas solo quedó una fuera del análisis factorial confirmatorio que corresponde a la pregunta 48: cómo fija el precio de sus mercancías o servicios. Por otra parte, este paso del análisis nos permite además, a través de la evaluación de las ponderaciones, identificar qué variables son las de mayor representatividad que serán las incluidas en el IGEF y aquellas que definitivamente no aportan y también quedarán eliminadas del estudio. Considerando cada una de las puntuaciones, se toma como referencia aquellas que están por encima de 0.4 , especificando que las que se encuentran entre 0.5 y 0.7 son significativas y las mayores a 0.7 se consideran relevantes. En esta evaluación se observa que del total de variables solo una quedó eliminada, la que hace referencia al destino que se le dio al préstamo ( $P-89)$, del resto se obtuvieron 4 relevantes, 3 significativas y una de menor aportación lo que ratifica y valida la selección de variables obtenidas.

Una vez determinado los factores, se procede a la construcción del IGEF, donde se utiliza como ponderaciones, el porcentaje de varianza explicado. En este punto los valores reflejan que entre los tres factores se logra explicar aproximadamente el 51\% (23.8 f1, $14.9 \mathrm{f2}, 12.1 \mathrm{f} 3)$ de la varianza total. Con este resultado, aplicando la fórmula 1 expresada en la metodología se procede a la obtención del IGEF, el cual deberá ser estandarizado aplicando la fórmula 2. Posteriormente se realiza un histograma de frecuencia para obtener diferentes rangos de clases del IGEF y poder determinar y evaluar cómo se reflejan a través del mismo, los niveles de gestión que muestran las microempresas estudiadas. El cálculo del histograma de frecuencia, arrojó para la muestra de estudio, un total de 13 rangos de clase. Para cada intervalo fueron determinadas las frecuencias considerando los límites inferiores y superiores lo cual se puede observar en la cuadro 2. Es válido destacar que en los rangos del 10 al 12 no quedó incluida ninguna de las microempresas de la muestra.

Con esta información podemos dilucidar que el mayor porcentaje de las microempresas analizadas se encuentran en los rangos inferiores, específicamente pudiéramos decir que el $70 \%$ correspondiente a 1894 microempresas de la muestra total, se encuentran ubicadas en el rango de 0 a 0.16 , es decir los intervalos 1 y 2 . Esto nos demuestra que la mayoría de las microempresas analizadas tienen un índice con valores cercanos a 0, específicamente por debajo de 0.16. También se extrae del análisis que existen 120 microempresas entre 0.24 y 0.48 , las 12 restantes reflejan niveles de gestión más elevados que el resto encontrándose la mayoría en rangos superiores a 0.5 y cercanos a la unidad. Este análisis da evidencias del nivel de GEF que manejan la mayor parte de las microempresas estudiadas, pero para poder complementar este resultado con el impacto que tiene el índice sobre los resultados empresariales, separamos los ejercicios de regresión en dos momentos, una primera regresión (cuadro 3 ) con el INGEF y un segundo ejercicio (cuadro 4) con el IGEF por ran-

\begin{tabular}{|cccccc|}
\hline \multicolumn{5}{c}{ Cuadro 2. Cálculo de frecuencias por rangos de clase. } \\
$\begin{array}{c}\text { No de } \\
\text { intervalos }\end{array}$ & $\begin{array}{c}\text { Límite } \\
\text { inferior }\end{array}$ & $\begin{array}{c}\text { Límite } \\
\text { superior }\end{array}$ & Frecuencia & $\begin{array}{c}\text { Frecuencia } \\
\text { relativa }\end{array}$ & $\begin{array}{c}\text { Frecuencia } \\
\text { acumulada }\end{array}$ \\
\hline 1 & 0.00 & 0.08 & 436 & 0.16 & 0.16 \\
\hline 2 & 0.08 & 0.16 & 1458 & 0.54 & 0.70 \\
\hline 3 & 0.16 & 0.24 & 671 & 0.25 & 0.95 \\
\hline 4 & 0.24 & 0.32 & 100 & 0.04 & 0.99 \\
\hline 5 & 0.32 & 0.40 & 15 & 0.01 & 1.00 \\
\hline 6 & 0.40 & 0.48 & 5 & 0.00 & 1.00 \\
\hline 7 & 0.48 & 0.56 & 4 & 0.00 & 1.00 \\
\hline 8 & 0.56 & 0.65 & 1 & 0.00 & 1.00 \\
\hline 9 & 0.65 & 0.73 & 1 & 0.00 & 1.00 \\
\hline 10 & 0.73 & 0.81 & 0 & 0.00 & 1.00 \\
\hline 11 & 0.81 & 0.89 & 0 & 0.00 & 1.00 \\
\hline 12 & 0.89 & 0.97 & 0 & 0.00 & 1.00 \\
\hline 13 & 0.97 & 1.00 & 1 & 0.00 & 1.00 \\
\hline
\end{tabular}

Fuente: Elaboración Propia con base al Histograma de Frecuencia.

gos de clase. Es importante acentuar que en las regresiones que se realizan, las variables utilizadas en el análisis factorial quedarán representadas a través del índice ya que éstas fueron utilizadas para su construcción. Una vez aclarado esto, aplicando la metodología con las variables incorporadas al modelo, en este caso la variable dependiente que representa los resultados empresariales y las variables independientes que están conformadas por capital, trabajo, el IGEF, el sexo y la edad, se obtienen los siguientes resultados:

\begin{tabular}{cccc}
\multicolumn{4}{c}{ Cuadro 3. Resultados de la regresión con el IGEF. } \\
$\begin{array}{c}\text { Variables } \\
\text { del Modelo }\end{array}$ & $\begin{array}{c}\text { Coeficientes } \\
\text { estandarizados }\end{array}$ & $t$ & Sig. \\
Constante & Beta & $B$ & Errortíp. \\
IGEF & & -0.666 & 0.506 \\
Capital & 0.245 & 13.655 & 0.000 \\
Trabajo & 0.275 & 15.551 & 0.000 \\
Sexo & 0.053 & 3.089 & 0.002 \\
Edad & 0.166 & 9.607 & 0.000 \\
R cuadrado $\quad \begin{array}{c}\text { Error típ. de la } \\
\text { estimación }\end{array}$ & -0.03 & -1.743 & 0.081 \\
$0.237 \quad$ DW & 1.987 & 166.494 & $.000(a)$
\end{tabular}

Fuente: Elaboración Propia en base al procesamiento de datos en el paquete estadistico SPSS

Realizando una interpretación de la información se puede observar a través de las salidas estadísticas, la bondad de ajuste y la capacidad explicativa del modelo planteado, las cuales reflejan que el modelo aplicado es un modelo ajustado. En relación a las variables incorporadas, comenzando por el IGEF construido, se obtuvo que el impacto sobre los resul- 
tados es positivo y significativo a un nivel de significancia del $5 \%$. De igual forma las variables representativas de capital y trabajo resultaron ser positivas y significativas. Estos resultados muestran evidencia sustantiva de la relación de dependencia que existe entre el nivel de producción y la totalidad de los medios empleados para realizarla, donde se destaca que el IGEF es una de las variables del modelo que mayor impacto tiene sobre los cambios de la variable dependiente (las utilidades de la empresa). Se corrobora una vez más la hipótesis de que para que las empresas sean cada vez más productivas, una adecuada gestión económico-financiera en el uso eficiente de sus recursos, contribuirá a que la empresa esté en mejores condiciones para evolucionar hacia el incremento de sus utilidades, su progreso y desarrollo.

A este análisis se le adiciona el segundo ejercicio, utilizando el IGEF para cada rango de clase, donde los resultados muestran cómo, a medida que el IGEF va moviéndose de los niveles cercanos a 0 hacia la unidad, el impacto sobre los resultados se convierte de negativo a positivo. Estos resultados se pueden apreciar en la cuadro 4.

Analizando estos datos nos percatamos de que para los intervalos entre 0 y 0.161 el impacto del índice es negativo y significativo al $5 \%$, lo cual está en congruencia con los resultados que arrojó el histograma de frecuencia, donde en este mismo rango es donde se concentra la mayor cantidad de microempresas analizadas (70\%). El resto de las variables incluidas en el modelo, capital y trabajo, resultaron ser positivas y significativas, comportamiento que se mantiene de manera similar en el resto de los intervalos. Continuando con la interpretación de los datos, podemos percatarnos que a medida que el índice va tomando valores cada vez más cercanos a 1, el impacto se vuelve de negativo a positivo y continua siendo significativo exceptuando los intervalos entre 0.484 y 0.645 y el último, entre 0.726 y 1 donde resultaron ser no significativos ni al $5 \%$ ni al $10 \%$. Por otra parte destacar que el valor de las $\mathrm{R}$ cuadrado en la mayoría de los intervalos se encuentra alrededor de 0.18 y 0.19 , sin embargo en los intervalos de $0.242-0.323$ - 0.403 tomó valores de 0.235 y 0.21 respectivamente. Por tanto, considerando estos resultados pudiéramos revelar que la mayor parte de las microempresas analizadas manifiestan niveles inferiores en su GEF que afectan negativamente los resultados que ellas obtienen. Por otra parte, aquellas microempresas que se ubicaron en intervalos más altos que los anteriores con mejores índices de GEF, como las 120 microempresas que se encuentran en los intervalos de frecuencia cercanos a 0.4 y 0.5 , el impacto del índice es positivo.

\section{Conclusiones.}

Debido a la importancia y representatividad que han ido ganando las microempresas dentro de los estratos empresariales de México, la presente investigación se realizó con el fin de aportar nuevos conocimientos y llegar a conclusiones que enriquezcan la disponibilidad de información con la que se cuenta actualmente acerca de cómo funcionan este tipo de empresas, qué característica las distinguen del resto, cómo realizan sus actividades y qué factores de mejora se pudieran complementar para que estas puedan desarrollarse y crecer. Por tanto el objetivo de este estudio se concentró en evaluar el nivel de gestión económico-financiera que se aplica en las microempresas mexicanas a partir de la elaboración de un índice que permitiera a su vez, determinar el impacto que tiene esa gestión sobre sus resultados empresariales. Para ello se consultó la literatura que conforma la teoría sobre el tema, se investigó acerca de los estudios precedentes y los resultados obtenidos y de igual forma se aplicaron diversas metodologías que contribuyeron a la obtención de los resultados.

La investigación nos permite concluir en primera instancia que la gestión económico-financiera es una actividad que tiene una fuerte influencia en el destino de las empresas, pues incluye tomar decisiones en áreas que son elementales para la permanencia de estas en los mercados. Las hipótesis planteadas pudieron ser comprobadas a partir de la revisión de las teorías acerca de la temática y de aplicación de las herramientas metodológicas. A través del análisis factorial se confirmó la $\mathrm{H} 1$ ya que las variables seleccionadas para la con-

\begin{tabular}{|c|c|c|c|c|c|c|c|c|c|c|c|c|c|c|c|c|c|c|c|c|}
\hline \multicolumn{21}{|c|}{ Cuadro 4. Resultados de la regresión con el IGEF por rangos de clase } \\
\hline Rango & \multicolumn{2}{|c|}{$0-.081$} & \multicolumn{2}{|c|}{$.081-.161$} & \multicolumn{2}{|c|}{$.161-242$} & \multicolumn{2}{|c|}{$.242-.323$} & \multicolumn{2}{|c|}{$.323-403$} & \multicolumn{2}{|c|}{$.403-.484$} & \multicolumn{2}{|c|}{$.484-.565$} & \multicolumn{2}{|c|}{$.565-.645$} & \multicolumn{2}{|c|}{$.645-.726$} & \multicolumn{2}{|c|}{$.726-1.0$} \\
\hline Modelo & Beta & Sig & Beta & Sig & Beta & Sig & Beta & Sig & Beta & Sig & Beta & Sig & Beta & Sig & Beta & Sig & Beta & Sig & Beta & Sig \\
\hline (Constante) & & 0.0 & & 0.0 & & 0.0 & & 0.0 & & 0.0 & & 0.0 & & 0.0 & & 0.0 & & 0.0 & & 0.0 \\
\hline IGEF & -0.0 & 0.0 & -0.1 & 0.0 & 0.0 & 0.0 & 0.2 & 0.0 & 0.1 & 0.0 & 0.0 & 0.0 & 0.0 & 0.1 & 0.0 & 0.8 & 0.0 & 0.0 & 0.0 & 0.9 \\
\hline Capital & 0.3 & 0.0 & 0.3 & 0.0 & 0.3 & 0.0 & 0.2 & 0.0 & 0.3 & 0.0 & 0.3 & 0.0 & 0.3 & 0.0 & 0.3 & 0.0 & 0.3 & 0.0 & 0.3 & 0.0 \\
\hline Trab. & 0.0 & 0.0 & 0.0 & 0.0 & 0.0 & 0.0 & 0.0 & 0.0 & 0.0 & 0.0 & 0.0 & 0.0 & 0.0 & 0.0 & 0.0 & 0.0 & 0.0 & 0.0 & 0.0 & 0.0 \\
\hline Sexo & 0.2 & 0.0 & 0.1 & 0.0 & 0.1 & 0.0 & 0.1 & 0.0 & 0.1 & 0.0 & 0.2 & 0.0 & 0.2 & 0.0 & 0.2 & 0.0 & 0.2 & 0.0 & 0.2 & 0.0 \\
\hline Edad & -0.0 & 0.2 & -0.0 & 0.4 & -0.0 & 0.3 & -0.0 & 0.1 & -0.0 & 0.2 & -0.0 & 0.4 & -0.0 & 0.3 & -0.0 & 0.3 & -0.0 & 0.3 & -0.0 & 0.3 \\
\hline R cuadrado & 0.1 & & 0.1 & & 0.1 & 86 & 0.2 & 235 & 0.2 & 10 & 0. & 186 & 0. & 184 & 0.1 & 184 & 0.1 & 187 & 0.1 & 184 \\
\hline Durbin-Watson & 1.9 & & 1.9 & & 1.9 & 178 & 1.5 & 269 & 1.9 & 180 & & 973 & 1.5 & 973 & 1.5 & 973 & 1.9 & 972 & & 973 \\
\hline
\end{tabular}

Fuente: Elaboración propia a partir del procesamiento de datos en el paquete estadístico SPSS. 
formación del IGEF fueron validadas por esta herramienta. A su vez los elementos que fueron utilizados en la conformación del índice están en correspondencia con estudios precedentes como los de Correa y Jaramillo (2007), Escobar, Arias y Portilla (2009), Aguilar, Ramírez y Barrón (2007) y Mungaray, et al. (2007), donde de igual forma fueron incluidos por estos investigadores como parte de sus comprobaciones, pero a diferencia de ellos, la presente investigación tiene como aportación principal la evaluación de la GEF de manera integradora aplicando la metodología de creación del índice, ya que las investigaciones empíricas que le anteceden, utilizan enfoques más dirigidos a la gestión financiera, además de que las técnicas utilizadas o propuestas se basan en otros métodos y procedimientos distintos al que aquí se construye.

Por otra parte, los resultados que arroja este estudio permiten conocer de una manera integrada que cuando las microempresas manejan niveles elevados de GEF esto les impacta positivamente en sus resultados, quedando comprobada de esta manera la $\mathrm{H} 2$. En este sentido, estamos haciendo referencia a que en la medida en que sean capaces de incorporar en su gestión y llevar adecuadamente las decisiones de inversión en recursos, las decisiones de financiación, distribución, asignación de recursos y el manejo de los mismos de forma tal que se obtengan los niveles de rentabilidad esperados así como la obtención de utilidades, todo ello acompañado de la contabilidad como fuente de información primaria, entonces estarán contribuyendo a que sus resultados sean cada vez mayores.

Por tanto, es importante que las instituciones y organismos gubernamentales encargados de promover e impulsar el crecimiento y desarrollo de estas empresas, establezcan políticas públicas, normativas, o programas, que estén orientados a optimizar sus procesos de gestión, a mejorar el aprovechamiento de sus recursos económicos y financieros, así como brindar mayores oportunidades para la obtención de financiamientos que estén acorde con sus niveles de generación de ingresos y utilidades.

A pesar de la contribución positiva de este estudio, no podemos dejar de mencionar las limitaciones que presenta el mismo, las cuales están asociadas en mayor medida con la escasez de información existente respecto a las microempresas. Es conocido y así se ha manifestado en diversos espacios, que existe un número reducido de bases de datos que contemplen diversidad de elementos sobre la funcionalidad de las microempresas, por lo que esto pudiera ser una oportunidad viable para futuras investigaciones. Tomando en consideración que la base de datos utilizada para el estudio no fue confeccionada por los investigadores, sino que hubo que tomar variables proxys de la ya existente, se pudiera confeccionar y aplicar un instrumento específico para el análisis donde incluso, el número de observaciones podría ser mucho más abarcador. Por otra parte pudiera aplicarse la metodología aquí propuesta a partir de la creación del índice, para medir el nivel de gestión en las microempresas pero, en esta ocasión, clasificadas por sectores de actividad económica.

\section{Referencias}

Aguilar J., Ramírez N., Barrón K. (2007). Conformación de la microempresa marginada en la frontera norte de México. Estudios Fronterizos, 8 (15), 51-71.

Amat, J. Ma. (1992). El Control de Gestión: Una perspectiva de Dirección. Barcelona, España: Ed. Ediciones Gestión 2000 S.A.

Block, S. B. y Hirt, G.A. (2013). Fundamentos de administración Financiera. (14a. ed.). Ciudad de México, México: Editorial McGraw-Hill Interamericana.

Brealey, R. A. y Myers, S. C. (2010). Principios de finanzas corporativas. (9a. ed.).Ciudad de México, México: Editorial McGraw-Hill Interamericana.

Correa, J.A. y Jaramillo, F. (2007). Una aproximación metodológica y prospectiva a la gestión financiera en las pequeñas empresas. Contaduría Universidad de Antioquia, (50), 93-118.

Dahmen, P. \& Rodríguez, E. (2014). Financial Literacy and the Success of Small Businesses: An Observation from a Small Business Development Center. Numeracy, 7 (1), 1-12.

Escobar, G.E.; Arias, L. y Portilla, L.M. (2009). Modelo para medir la situación financiera en empresas pyme. Scientia Et Technica, Universidad Tecnológica de Pereira, Colombia, XV (43), 273-278.

Keat, P. G. y Young, P. K. Y. (2004). Economía de la empresa. (4 ed.). Madrid, España: Pearson Prentice Hall.

Lawrence, J.G. (2007). Principios de Administración Financiera. (11 ed.). Ciudad de México, México: Pearson Educación.

Mariño B., Gladys Y. y Medina S. (2009). La administración financiera: una utopía en las microempresas. Colombia. Criterio Libre, 7 (11), 123-144.

Mazzarol, T., Reboud, S. \& Clark, D. (2015). The financial management practices of small to medium enterprises. Small Enterprise Association of Australia and New Zealand 28 th Annual SEAANZ Conference Proceedings 1-3 July Melbourne, 2015, p 1-22.

Mungal, A. \& Garbharran, H. L. (2014). Cash Management Challenges of Small Businesses in a Developing Community. Mediterranean Journal of Social Sciences MCSER Publishing, 5 (27), 11-21. 
Mungaray, A., Ocegueda, J.M., Ledezma, D., Ramírez, N., Ramírez, N., y Alcalá, C. (2007). Formación por medio del servicio. Un modelo de servicio social universitario en apoyo a microempresas marginadas. Revista El trimestre económico, 74 (296), 987-1011.

Ochoa, G. A. y Saldívar del Ángel, R. (2012). Administración financiera correlacionada con las NIF (3a. ed. Ciudad de México, México, Editorial McGraw-Hill Interamericana.

Paramasivan C., Subramanian T. (2009). Financial Management. New Delhi, India: New Age International Pvt. Ltd., Publishers.

Pavón, L. (2010). Financiamiento a las microempresas y las pymes en México 2000-2009. Unidad de Estudios del Desarrollo, Agencia Española de Cooperación Internacional para el Desarrollo. Santiago de Chile, Chile: Editorial: CEPAL Colección: Series de la CEPAL (p. 68).

Ramírez. M., Mungaray. A., y Guzmán, N. (2009). Restricciones de liquidez en microempresas y la importancia del financiamiento informal en Baja California. Región y Sociedad, 21 (44), 71-90.

Rathnasiri, U.A.H.A. (2015). The Financial Management Practices of Small and Medium Enterprises in Sri Lanka. Global Journal of Contemporary Research in Accounting, Auditing and Business Ethics (GJCRA), 1 (2), 374-399.
Rodríguez, J. (2010). Administración de pequeñas y medianas empresas. (6 ed.). Ciudad de México, México: Cengage Learning.

Ross, S. A., Westerfield, R. W. y Jaffe, J. F. (2012). Finanzas corporativas. (9a. ed.). Ciudad de México, México: Editorial McGraw-Hill Interamericana.

Santiesteban Z., Fuentes, F., Leyva C. y Lozada, N. (2009, mayo). Tecnología para el análisis de la Rentabilidad Económica. Una aproximación a la eficiencia empresarial. Contribuciones a la Economía. http://www.eumed.net/ ce/2009a/zfen.htm

Simpson, S.D. (2011 ). The Difference Between Finance And Economics. CFA on April 21. http://www.investopedia. com/articles/economics/11/difference-between-financeand-economics.asp

Solomon, T. (2014). An assessment of financial management practices of small businesses: case study of Sokoru District, (Tesis de Maestría) College of Business and Economics, Jimma University, Ethiopia.

Suárez, D.C. (2003). Conceptos y formulación de indicadores. Manizales, Colombia: Banco Interamericano de DesarroIlo. Universidad Nacional de Colombia - Sede Manizales. Instituto de Estudios Ambientales Indicadores de Gestión de Riesgos. BID - CEPAL - IDEA.

Van, J.C. (1995). Administración Financiera. Ciudad de México, México. Compañía Editorial Continental SA de CV. 